\title{
Electron temperature of Titan's sunlit ionosphere
}

\author{
M. Galand, ${ }^{1}$ R. V. Yelle, ${ }^{2}$ A. J. Coates ${ }^{3}$ H. Backes, ${ }^{4}$ and J.-E. Wahlund ${ }^{5}$ \\ Received 20 July 2006; revised 8 September 2006; accepted 2 October 2006; published 1 November 2006.
}

[1] Titan's upper atmosphere is ionized by solar radiation and particle bombardment from Saturn's magnetosphere. The induced ionosphere plays a key role in the coupling of Titan's atmosphere with the Kronian environment. It also provides unique signatures for identifying energy sources upon Titan's upper atmosphere. Here we focus on observations from the first, close flyby by the Cassini spacecraft and assess the ionization and electron heating sources in Titan's sunlit ionosphere. We compare CAPS electron spectra with spectra produced by an electron transport model based on the INMS neutral densities and a MHD interaction model. In addition, we compare RPWS electron temperature against the models. The important terms in the electron energy equation include loss through excitation of vibrational states of $\mathrm{N}_{2}$ and $\mathrm{CH}_{4}$, Coulomb collisions with suprathermal electrons, and thermal conduction. Our analysis highlights the important role of the magnetic field line configuration for aeronomic studies at Titan. Citation: Galand, M., R. V. Yelle, A. J. Coates, H. Backes, and J.-E. Wahlund (2006), Electron temperature of Titan's sunlit ionosphere, Geophys. Res. Lett., 33, L21101, doi:10.1029/2006GL027488.

\section{Introduction}

[2] The Earth's strong magnetic field constrains the movement of electrons, determining how different locations in the upper atmosphere are coupled through transport of energy and suprathermal electrons. Presumably, the same is true for the Jovian planets, though we have no direct measurements for these atmospheres. Venus and Mars are characterized by complex magnetic environments, determined by the interaction of their ionospheres and the solar wind. Depending on the solar activity, the ionosphere of these two planets can be significantly magnetized. At Mars the presence of a crustal magnetic field is an additional source of complexity to the magnetic field line structure and the solar wind-ionosphere interaction. The magnetic environment of Titan is also complex, caused by the interaction of Titan's ionosphere with Saturn's magnetosphere. The field is strong enough to have a controlling influence on the aeronomy of Titan's upper atmosphere. We show below that the magnetic structure in Titan's upper atmosphere has a determining effect on the electron temperature and on the

\footnotetext{
${ }^{1}$ Department of Physics, Imperial College, London, UK.

${ }^{2}$ Department of Planetary Sciences, University of Arizona, Tucson, Arizona, USA

${ }^{3}$ Mullard Space Science Laboratory, University College London, Dorking, UK.

${ }^{4}$ Klinikum der Universtität zu Köln, Cologne, Germany.

${ }^{5}$ Swedish Institute of Space Physics, Uppsala, Sweden.
}

Copyright 2006 by the American Geophysical Union. 0094-8276/06/2006GL027488\$05.00 transport and distribution of suprathermal electrons, and therefore will also affect ionization rates, excitation rates, neutral heating rates, and other key aeronomical processes.

[3] Flybys through Titan's upper atmosphere of the comprehensive payload onboard the Cassini spacecraft offer us a unique opportunity to identify the energy sources upon Titan's ionosphere. Our investigation of this system combines data from four Cassini instruments using as a binding element a comprehensive set of electron kinetic and fluid interaction models. Such a well-constrained problem allows us to infer the major ionization and heating sources and to assess the energy balance in Titan's ionosphere.

[4] In this paper, we focus on the sunlit part of the first, close flyby of Titan by Cassini, referred as "TA" in the Cassini project terminology. The good agreement of the suprathermal electron intensities and ionospheric electron temperatures between the observations and the model shows that the major energy source at the two times studied was solar irradiance. Photoelectron signatures identified in both the computed and measured electron spectra corroborate this finding. Moreover, the observed ionospheric electron temperatures and the isotropic nature of the observed electron spectra cannot be understood without taking into account the complex draping of the magnetic field line around Titan.

[5] The relative importance of energy sources of solar and magnetospheric origin varies with the local time at Titan and the location of Titan within Saturn's magnetosphere [e.g., Nagy and Cravens, 1998]. Previous modeling studies focused on the energetics in Titan's ionosphere including both solar and magnetospheric particle heating. Gan et al. [1992] and Keller et al. [1992] computed the electron transport and electron energy equations coupled with a photochemical ionospheric model along a magnetic field line assumed to be a parabola around Titan. Roboz and Nagy [1994] solved the coupled continuity, momentum, and energy equations for ionospheric electrons and ions and the electron transport equation. They assumed a radial magnetic field line in the wake side and introduced correction factors on the thermal conductivities to take into account horizontal magnetic fields, in the subram region. The importance of the magnetic field geometry was pointed out in these studies, but the lack of observational constraints limited these efforts. Thanks to the rich observational data set provided by Cassini, we can extend these first attempts for a well-constrained estimate of the energy balance in Titan's ionosphere.

\section{Cassini Setting}

[6] The TA flyby of Titan by the Cassini spacecraft occurred on October 26, 2004, while Titan was close to southern hemisphere summer solstice. The solar declination was $-23^{\circ}$. The $\mathrm{F}_{10.7}$ solar index was $137 \times$ $10^{-22} \mathrm{Wm}^{-2} \mathrm{~Hz}^{-1}$. Titan was located at 10.6 hours Saturn 


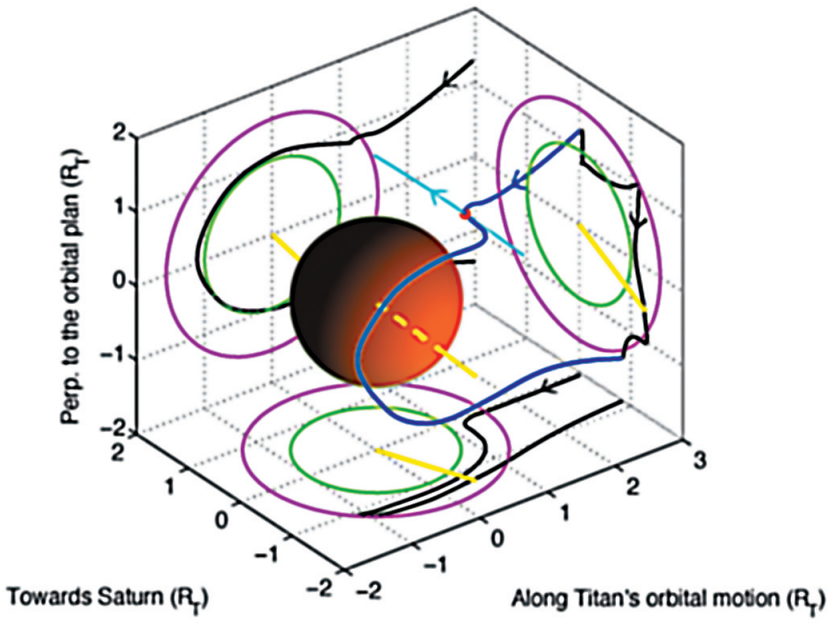

Figure 1. 3D representation of the modeled magnetic field line (blue) crossing the location of Cassini (red dot) at $-100 \mathrm{~s}$ from CA during the TA flyby. The projections of the magnetic field line are shown in black. The image of Titan is courtesy NASA/JPL-CalTech. The projections of Titan's body are shown in green. The exobase at an altitude of $1450 \mathrm{~km}$ is shown in magenta and the Sun's direction, in yellow. Cassini trajectory is represented in cyan.

local time. The Cassini spacecraft flew from dayside to nightside through the magnetic wake, which leads Titan in its orbit. During the inbound path of the flyby, the component of the magnetic field along Titan's orbital motion measured by the MAG instrument [Dougherty et al., 2004] was negative [Backes et al., 2005]. Energetic electrons with pitch angles greater or lesser than $90^{\circ}$ were moving upward or downward, respectively.

[7] We have selected two times of interest during which Cassini was crossing the sunlit region of Titan's ionosphere near closest approach (CA): $-100 \mathrm{~s}$ and $-200 \mathrm{~s}$ from CA. The location of Cassini was characterized as follows: an altitude of $1219 \mathrm{~km}(1352 \mathrm{~km})$, a latitude of $36^{\circ}\left(32^{\circ}\right)$, a local time of 16.0 (15.4), and a solar zenith angle of $82^{\circ}$ $\left(74^{\circ}\right)$ at $-100 \mathrm{~s}(-200 \mathrm{~s})$ from CA. The MHD interaction model described in Section 3 predicts that the magnetic field lines crossing the Cassini trajectory at these times are draped around Titan's ionosphere (see Figure 1). The altitude and solar zenith angle variations along the magnetic field line crossed by the spacecraft at $-100 \mathrm{~s}$ are shown in Figure 2. The configuration at $-200 \mathrm{~s}$ is very similar and is thus not shown.

[8] Data from the Electron Spectrometer sensor (ELS), a sub-system of the Cassini Plasma Spectrometer (CAPS) [Young et al., 2004], and the Langmuir Probe (LP), a subsystem of the Cassini Radio and Plasma Wave Science (RPWS) [Gurnett et al., 2004], are used in this study for direct comparison with models. The ELS measures energetic electrons, providing the electron distribution at energies from $0.6 \mathrm{eV}$ to $28 \mathrm{keV}$ at a spectral resolution of $17 \%$ and an angular resolution of $20^{\circ}$. Correction for the spacecraft potential as determined from CAPS ion species identification [Crary et al., 2006] has been applied to the electron distributions. Among other parameters, the LP measures the characteristics of thermal electrons, including the electron density and temperature [Wahlund et al., 2005].

\section{Electron Kinetic and Fluid Models}

[9] The transport of the suprathermal electrons in Titan's upper atmosphere is modeled with a multi-stream code solving the Boltzmann equation [Galand et al., 1999]. Redistribution in angle occurs through elastic collisions with atmospheric neutrals, while energy degradation occurs through excitation and ionization of the neutrals and through Coulomb collisions with the thermal electrons. Angular redistribution through magnetic mirroring is not considered here. While a two-stream approach is sufficient for estimating angle-integrated quantities, a multi-stream approach provides the angular distribution of the electrons over a wide range of angles (here 16) - within the uncertainties in the differential cross sections. This computed angular distribution can be compared with that measured by CAPS/ELS (Section 4). Recent upgrades of the transport model include the use of $\mathrm{N}_{2}$ photo-ionization cross section of Samson et al. [1987] and Stolte et al. [1998] and of $\mathrm{CH}_{4}$ electron-impact ionization cross section of Liu and Shemansky [2006]. The solar flux, only incident energy source considered, is based on the solar EUV flux model for Aeronomic Calculations [Richards et al., 1994a, 1994b], scaled to the location of Titan.

[10] The temperature of the ionospheric electrons is derived from the solution of the time-independent electron energy equation including heating and cooling processes, and heat transfer through thermal conduction. Heating of ionospheric electrons is due solely to Coulomb collisions with suprathermal electrons, using the calculated energetic electron distribution and the formulation of Swartz et al. [1971]. The energy grid used to compute the electron spectrum and derived heating rates is logarithmic with 200 levels and a low energy limit of $0.1 \mathrm{eV}$. The cooling sources are electron impact of $\mathrm{N}_{2}$ and $\mathrm{CH}_{4}$, including vibrational and rotational excitation and elastic collisions [Banks and Kockarts, 1973; Gan and Cravens, 1992]. The thermal conductivity corrected for partially ionized gas is taken from Schunk and Nagy [2000]. The electron energy equation is solved applying an implicit method. No heat

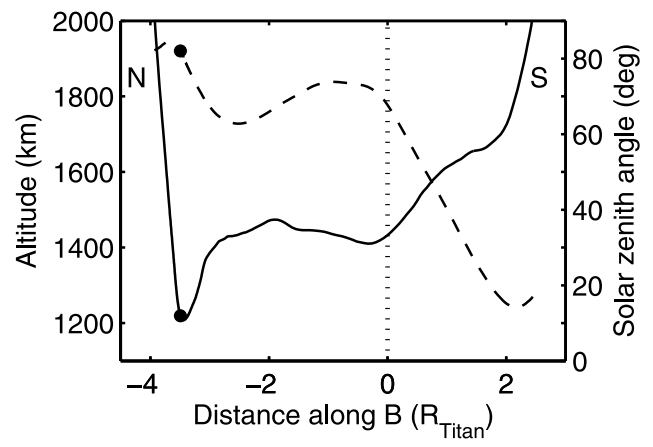

Figure 2. Altitude (solid line) and solar zenith angle (dashed line) as a function of the distance along the magnetic field line for $-100 \mathrm{~s}$ from CA. The distance has been arbitrarily taken negative in the northern hemisphere $(\mathrm{N})$ and positive in the southern $(\mathrm{S})$. The location of Cassini is shown with a dot. 
flow is assumed at the boundaries taken at an altitude of $2000 \mathrm{~km}$.

[11] Both kinetic and fluid calculations are carried out along a magnetic field line of variable geometry (see Figure 1) inferred from a 3D model describing Titan's interaction with the Saturnian magnetospheric plasma [Backes, 2004] and applied to the TA flyby solar conditions. The ideal magnetohydrodynamic (MHD) equations have been extended in order to take into account the effects of Titan's dense neutral atmosphere on the plasma interaction. The magnetic, upstream conditions assumed as input to the MHD model are based on Voyager observations, where the Saturnian magnetic field vector is perpendicular to Titan's orbital plane. In order to fit the magnetic, upstream conditions observed by the MAG instrument onboard Cassini, Backes et al. [2005] applied a rotation of $23.8^{\circ}$ towards Saturn to the calculated magnetic field lines and ionospheric structure. Such a rotation shifts the location of the dayside ionosphere, resulting in a different direction of the Sun. However, close to Titan, the structure of the magnetic field line is more sensitive to the ionospheric conditions, and hence here the solar direction, than to the magnetic, upstream conditions. Therefore, we have not applied this rotation in the present study. The optimum scenario would be to redo the MHD simulations assuming both the correct TA solar direction and the magnetic, upstream conditions observed by MAG. Such a run will not be available with this MHD model in the foreseeable future, but the uncertainty introduced by the present approximation does not alter the main findings of this study.

[12] The electron transport and the electron energy models depend on the altitude profiles of $\mathrm{N}_{2}$ and $\mathrm{CH}_{4}$ densities inferred from the Ion and Neutral Mass Spectrometer (INMS) measurements onboard Cassini during the TA flyby [Waite et al., 2005; Yelle et al., 2006]. Assuming hydrostatic equilibrium and solving the diffusion equation for a binary gas mixture in a gravitational field, a fit of the model to the $\mathrm{N}_{2}$ density provides an estimate of the altitude profile of the neutral temperature [Yelle et al., 2006], used as input of the electron energy equation. Such a model was also used to extrapolate the $\mathrm{N}_{2}$ and $\mathrm{CH}_{4}$ neutral density profiles towards altitudes below the altitude of CA. The electron density is estimated from the total neutral density and electron production rate using a parametrization based on the ionospheric model developed by Vuitton et al. [2006].

\section{Comparison Between Observations and Model}

[13] The electron spectra at $100 \mathrm{~s}$ and at $200 \mathrm{~s}$ before CA measured by the CAPS/ELS instrument (Section 2) are shown in Figures $3 \mathrm{a}$ and $3 \mathrm{~b}$. Eight samples of $2 \mathrm{~s}$ each are averaged together to improve the signal-to-noise ratio. The spatial range corresponding to the 8 averaged samples is about $100 \mathrm{~km}$. The electron spectra computed at the location of Cassini (Section 3) do not show any significant difference over the $16 \mathrm{~s}$ duration. Between $8 \mathrm{eV}$ and $55 \mathrm{eV}$, the agreement in magnitude between the observed and calculated electron spectra is better than a factor of 3.5 at $-100 \mathrm{~s}$ and of 4.5 at $-200 \mathrm{~s}$ (Figures $3 \mathrm{a}$ and $3 \mathrm{~b}$ ).

[14] Unique signatures of photoelectrons are identified in both the observed and modeled spectra. The observed spectra show a peak near $24 \mathrm{eV}$ that is associated with
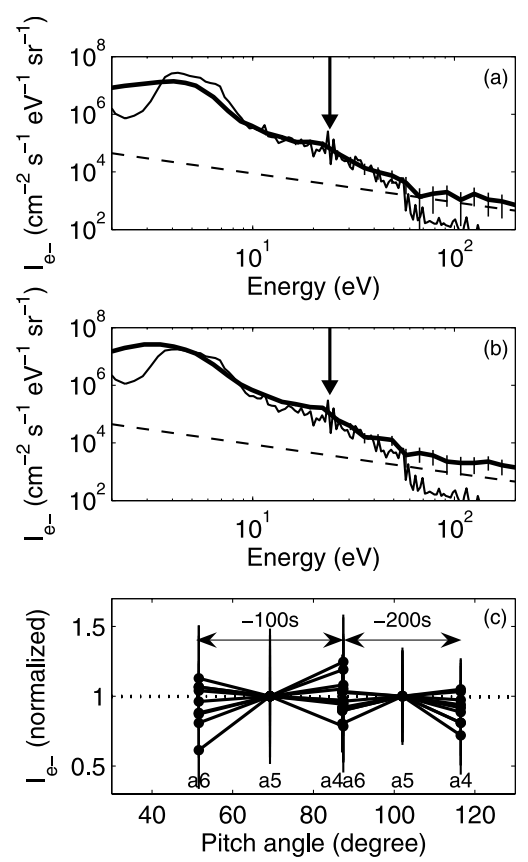

Figure 3. (a) Electron intensity as a function of the electron energy, observed by CAPS/ELS (thick, solid line), and modeled (thin, solid line) at $-100 \mathrm{~s}$ from CA. The measured spectrum is the average of 8 samples $(16 \mathrm{~s})$ and is associated with a pitch angle of $\sim 87^{\circ}$. The vertical bars represent the Poisson statistical errors. The dashed line is associated with the 1-count level averaged over 8 samples $(16 \mathrm{~s})$. The arrow corresponds to the energy of a photoelectron produced by ionization of $\mathrm{N}_{2}$ by $\mathrm{HeII}(30.4$ nm) solar photons to produce $\mathrm{N}_{2}{ }^{+}$in the A state. (b) Same but for $-200 \mathrm{~s}$ from CA. The pitch angle range for CAPS/ ELS is $101-132^{\circ}$. (c) Distributions in pitch angle of the energetic electrons normalized at the pitch angle associated with anode 5. The CAPS/ELS distributions are shown in solid line for anodes 4,5 , and 6 . The distributions are shown for two times: $-100 \mathrm{~s}$ from CA (anode 5 near $70^{\circ}$ ) and $-200 \mathrm{~s}$ from $\mathrm{CA}$ (anode 5 near $100^{\circ}$ ). At a given time, the various distributions are associated with energies ranging from 10 to $50 \mathrm{eV}$ and incremented by $5 \mathrm{eV}$. The vertical lines represent the Poisson statistical errors. The computed distributions over the 10 to $50 \mathrm{eV}$ range and at both $-100 \mathrm{~s}$ and $-200 \mathrm{~s}$ from $\mathrm{CA}$ are shown in dotted lines.

the ionization of $\mathrm{N}_{2}$ by the strong HeII (30.4 nm) solar line. Such an ionization may leave the $\mathrm{N}_{2}^{+}$ion in the $\mathrm{X}, \mathrm{A}$, or $\mathrm{B}$ state (as seen in the modeled spectra). For reference, the photoelectron energy $(24.1 \mathrm{eV})$ produced by the ionization of $\mathrm{N}_{2}$ into the dominant A state is shown with an arrow.

[15] The second signature of photoelectrons is the sharp decrease seen in both the modeled and observed electron spectra near $60 \mathrm{eV}$. It is induced by the drop in the solar spectrum near $16 \mathrm{~nm}$. The smaller decrease found in the ELS compared with the modeled spectra may be explained by the lower spectral resolution of the data set along with the significant statistical error near $60 \mathrm{eV}$.

[16] Above $60 \mathrm{eV}$ the comparison between the modeled and the measured spectra at $-100 \mathrm{~s}$ cannot be carried out due to too low count level. At -200 s however, the CAPS/ 
ELS spectrum exhibits a high energy tail. It is statistically significant but its close proximity to the one-count level makes a quantitative estimate difficult. Energetic electrons precipitating from Saturn's magnetosphere are the most likely source of this high energy tail. Their presence would yield the production of secondary electrons of lower energies and explain the slight underestimation of the computed electron spectrum (solely induced by solar photons) compared with the observed one below $60 \mathrm{eV}$.

[17] Below $8 \mathrm{eV}$ the observed and computed spectra differ. The dip seen between 2 and $4 \mathrm{eV}$ in the modeled spectra is produced by vibrational excitation of $\mathrm{N}_{2}$. It is predicted by electron transport model in the $E$-region terrestrial ionosphere [e.g., Nagy and Banks, 1970; Solomon et al., 2001]. However, such a distribution is unstable. Plasma instabilities induced by the gap are expected to occur, filling it in [Basu et al., 1982], but these are not included in the model.

[18] The measured and modeled suprathermal electron distributions are close to isotropic as illustrated in Figure 3c. Between 2 and $100 \mathrm{eV}$, the isotropy is satisfied by the modeled spectra over the whole angular range within $15 \%$. The most suitable anodes for quantitative inter-comparisons are anodes 4 to 6 , which are least affected by spacecraftplasma interaction effects. At $-100 \mathrm{~s}$ from CA, anodes 4, 5, and 6 over the 8 averaged samples cover pitch angles of $87-88^{\circ}, 69^{\circ}$, and $35-36^{\circ}$, respectively, while at $-200 \mathrm{~s}$, they cover $101-132^{\circ}, 88-115^{\circ}$, and $65-80^{\circ}$, respectively. The relative difference between the spectra of these three anodes is $50 \%$ at $-100 \mathrm{~s}$ over the 10 to $50 \mathrm{eV}$ range and $32 \%$ at $-200 \mathrm{~s}$ over the 10 to $100 \mathrm{eV}$ range. At energies larger than $60 \mathrm{eV}$ at $-100 \mathrm{~s}$, the large statistical error prevents any relevant comparison. The isotropy found in both the modeled and measured spectra attests to the local origin of the electron population. At both studied times, the Cassini spacecraft was located close to the minimum altitude along the magnetic field line (see Figure 2) associated with the maximum electron production rate.

[19] Figure 4 shows the computed electron temperatures along the magnetic field line (solid line) mapped versus altitude. The altitude mapping from the northern hemisphere (N) to the southern hemisphere (S) is illustrated in Figure 2. In the simulations the neutral temperature inferred from INMS observations (dotted line) (Section 3) is used as initial conditions for the electron temperature. Suprathermal electron heating raises the electron temperature from this initial assumption. As a response, the cooling rate, dependent on the difference between electron and neutral temperatures, increases. Thermal conduction along the magnetic field line redistributes energy in region of large electron temperature gradients. It is responsible for the constanttemperature profile found in the highest altitude regions.

[20] The value of the electron temperature derived from the RPWS/LP sensor (Section 2) along with the uncertainties estimated to be $\pm 15 \%$ are shown with crosses. For both $-100 \mathrm{~s}$ and $-200 \mathrm{~s}$, the computed value at the location of Cassini (dot) agrees very well with the observations within the error bars of the measurements. The electron temperature is fully explained by solar illumination only, with heating by photoelectrons and induced secondary electrons. Energetic sources from the magnetosphere, such as heat flow and particle precipitation, do not seem to play any
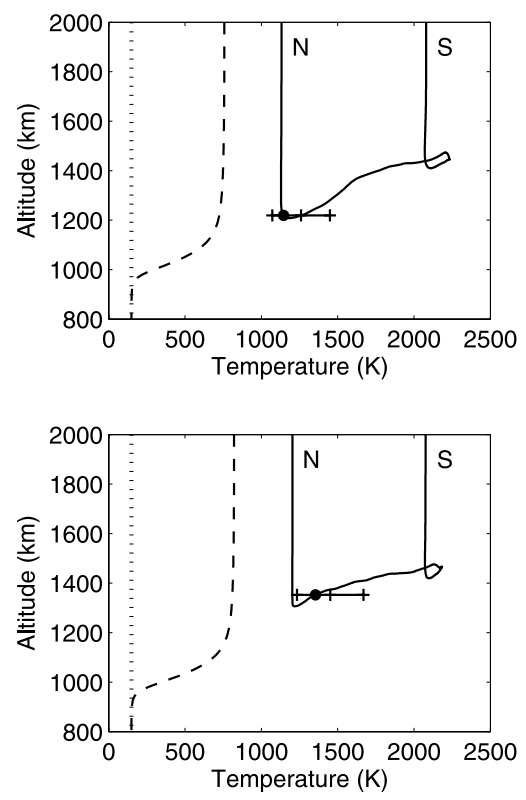

Figure 4. (top) Electron temperature computed along the magnetic field line mapped versus altitude (solid line) at $-100 \mathrm{~s}$ from CA. The location of Cassini along the magnetic field line is shown with a dot. The electron temperature measured by RPWS/LP at $-100 \mathrm{~s}$ from CA is represented as an horizontal bar with crosses. The central cross represents the effective value derived from the observations and the left and right crosses indicate the $\pm 15 \%$ uncertainty. Also shown are the altitude profile of the neutral temperature inferred from INMS during the TA flyby (dotted line) and the altitude profile of the electron temperature computed for a purely vertical field line passing through Cassini's location (dashed line). " $N$ " and "S" stand for northern and southern hemispheres, respectively. (bottom) Same but for $-200 \mathrm{~s}$ from CA.

significant role as a heating source of the sunlit ionosphere within $10-20 \%$ of the measurements - at $1200-1400 \mathrm{~km}$ during TA. They may become important at higher altitudes and on the nightside during TA.

\section{Discussion}

[21] Taking into account only solar photons as incident energy source upon Titan's upper atmosphere, the spectra of suprathermal electrons and the ionospheric electron temperatures computed in the model agree well in magnitude with the observations by CAPS/ELS and RPWS/LP, respectively, at $-100 \mathrm{~s}$ and $-200 \mathrm{~s}$ from CA. In addition, two unique signatures of photoelectrons were identified in both the modeled and the measured electron spectra: intensity peaks near $24 \mathrm{eV}$ and a decrease in electron intensity near $60 \mathrm{eV}$. These photoelectron signatures are present in the electron spectra previously predicted for Titan [Gan et al., 1992; Cravens et al., 2004]. They are also familiar features of the electron spectra at Earth observed from rockets and satellites and predicted by transport models [Nagy and Banks, 1970; Lee et al., 1980; Richards and Torr, 1988; Solomon et al., 2001].

[22] Our analysis also illustrates the important role the magnetic field line structure plays in interpreting Cassini 
observations in Titan's ionosphere. To illustrate this point we derive the electron spectrum and temperature profile assuming the magnetic field line to be along the local vertical passing through the location of Cassini. For such a case the maximum electron production rate is located well below the altitude of the spacecraft. The computed electron spectrum at the location of Cassini is anisotropic, with the maximum intensity found at $180^{\circ}$ pitch angle (upward). Over the pitch angle range covered by the CAPS/ELS anodes 4-6 at $-200 \mathrm{~s}$, the difference between modeled spectra computed along the vertical in the $10-100 \mathrm{eV}$ range is reaching 97\%, 3 times what was observed by CAPS/ELS (Section 4). The anisotropy found also contrasts with what is simulated when the complex structure of the magnetic field line is taken into account (see Figure 1 and Section 4). The electron temperature profile computed for a vertical field line configuration is shown in Figure 4 (dashed line). Below $\sim 1200 \mathrm{~km}$, thermal conduction towards lower altitudes yields a temperature value at the location of Cassini several hundreds of Kelvin smaller than the one derived from the simulation along a draped magnetic field line. The largely horizontal orientation of the field line at the location of Cassini (Figure 1) prevents energy to be conducted below. It is important to take this complex structure into account in order to be able to explain the isotropic suprathermal electron spectra observed by CAPS/ELS and the large temperature observed by RPWS/LP.

[23] Acknowledgments. We would like to thank J. Giacalone, D. Lummerzheim, and F. Neubauer very much for enriching discussions. We are grateful to S. Solomon for input regarding the solar model. RVY acknowledges the support of NASA grant NAG5-12699.

\section{References}

Backes, H. (2004), Titan's interaction with the Saturnian magnetospheric plasma, thesis, Inst. für Geophys. and Meteorol., Univ. of Cologne, Cologne, Germany.

Backes, H., et al. (2005), Titan's magnetic field signature during the first Cassini encounter, Science, 308,992-9995, doi: 10.1126/science.1109763. Banks, P. M., and G. Kockarts (1973), Aeronomy, Elsevier, New York.

Basu, B., T. Chang, and J. R. Jasperse (1982), Electrostatic plasma instabilities in the daytime lower ionosphere, Geophys. Res. Lett., 9, 68-71.

Crary, F. J., et al. (2006), Dynamics and composition of plasma at Titan, Science, in press.

Cravens, T. E., J. Vann, J. Clark, J. Yu, C. N. Keller, and C. Brull (2004), The ionosphere of Titan: An updated theoretical model, Adv. Space Res., 33, 212-215, doi:10.1016/j.asr.2003.02.012.

Dougherty, M., et al. (2004), The Cassini Magnetic Field investigation, Space Sci. Rev., 114, 331-383.

Galand, M., J. Lilensten, D. Toublanc, and S. Maurice (1999), The ionosphere of Titan: Ideal diurnal and nocturnal cases, Icarus, 140, $92-105$.

Gan, L., and T. E. Cravens (1992), Electron impact cross sections and cooling rates for methane, Planet. Space Sci., 40(11), 1535-1544.

Gan, L., C. N. Keller, and T. E. Cravens (1992), Electrons in the ionosphere of Titan, J. Geophys. Res., 97(A8), 12,137-12,151.
Gurnett, D. A., et al. (2004), The Cassini radio and plasma wave investigation, Space Sci. Rev., 114, 395-463.

Keller, C. N., T. E. Cravens, and L. Gan (1992), A model of the ionosphere of Titan, J. Geophys. Res., 97(A8), 12,117-12,135.

Lee, J. S., J. P. Doering, T. A. Potemra, and L. H. Brace (1980), Measurements of the ambient photoelectron spectrum from AI: II. AE-E measurements between 300 and $1000 \mathrm{~km}$ in solar minimum conditions, Planet. Space Sci., 28, 973-996.

Liu, X., and D. E. Shemansky (2006), Analysis of electron impact ionization properties of methane, J. Geophys. Res., 111, A04303, doi:10.1029/ 2005JA011454.

Nagy, A. F., and P. M. Banks (1970), Photoelectron fluxes in ionosphere, J. Geophys. Res., 75(31), 6260-6270.

Nagy, A. F., and T. E. Cravens (1998), Titan's ionosphere: A review, Planet. Space Sci., 9/10, 1149-1155.

Richards, P. G., and D. G. Torr (1988), Ratios of photoelectron to EUV ionization rates for aeronomic studies, J. Geophys. Res., 93(A5), 40604066.

Richards, P. G., J. A. Fennelly, and D. G. Torr (1994a), EUVAC: A solar EUV flux model for aeronomic calculations, J. Geophys. Res., 99(A5), $8981-8992$.

Richards, P. G., J. A. Fennelly, and D. G. Torr (1994b), Erratum: "EUVAC: A solar EUV flux model for aeronomic calculations"'[J. Geophys. Res., 99, 8981-8992 (1994)], J. Geophys. Res., 99(A7), 13,283-13,284.

Roboz, A., and A. F. Nagy (1994), The energetics of Titan's ionosphere, J. Geophys. Res., 99(A2), 2087-2093.

Samson, J. A. R., T. Masuoka, P. N. Pareek, and G. C. Angel (1987), Total and dissociative cross sections of $\mathrm{N}_{2}$ from threshold to $107 \mathrm{eV}, J$. Chem. Phys., 86, 6128-6132.

Schunk, R. W., and A. Nagy (2000), Ionospheres: Physics, Plasma Physics and Chemistry, Cambridge Atmos. and Space Sci. Ser., Cambridge Univ. Press, New York.

Solomon, S. C., S. M. Bailey, and T. N. Woods (2001), Effect of solar soft X-rays on the lower ionosphere, Geophys. Res. Lett., 28(11), 2149-2152.

Stolte, W. C., Z. X. He, J. N. Cutler, Y. Lu, and J. A. R. Samson (1998), Dissociative photoionization cross sections of $\mathrm{N}_{2}$ and $\mathrm{O}_{2}$ from 100 to 800 eV, At. Data Nucl. Data Tables, 69, 171-179.

Swartz, W. E., J. S. Nisbet, and A. E. S. Green (1971), Analytic expression for the energy transfer rate from photoelectrons to thermal electrons, J. Geophys. Res., 76(34), 8425-8426.

Vuitton, V., R. V. Yelle, and V. G. Anicich (2006), The nitrogen chemistry of Titan's upper atmosphere revealed, Astrophys. J., 647, L175L178.

Wahlund, J.-E., et al. (2005), Cassini measurements of cold plasma in the ionosphere of Titan, Science, 308,986-989, doi:10.1126/science.1109807.

Waite, H., et al. (2005), Ion Neutral Mass Spectrometer results from the first flyby of Titan, Science, 308, 982-986, doi:10.1126/science.1110652.

Yelle, R. V., et al. (2006), The vertical structure of Titan's upper atmosphere from Cassini Ion Neutral Mass Spectrometer measurements, Icarus, 182, 567-576, doi:10.1016/j.icarus.2005.10.029.

Young, D. T., et al. (2004), Cassini plasma spectrometer investigation, Space Sci. Rev., 114, 1-112.

H. Backes, Klinikum der Universität zu Köln, Cologne, Germany, (heback@web.de)

A. J. Coates, Mullard Space Science Laboratory, University College London, Holmburg St. Mary, Dorking RH5 6NT, UK. (ajc@mssl.ucl.ac.uk) M. Galand, Space and Atmospheric Physics Group, Department of Physics, Imperial College, Prince Consort Road, London SW7 2BW, UK. (m.galand@imperial.ac.uk)

R. V. Yelle, Department of Planetary Sciences, University of Arizona, Tucson, AZ 85721, USA. (yelle@lpl.arizona.edu)

J.-E. Wahlund, Swedish Institute of Space Physics, Uppsala Division, Box 537, SE-751 21 Uppsala, Sweden. (jwe@irfu.se) 